\title{
Review Article \\ Role of Myeloperoxidase in Patients with Chronic Kidney Disease
}

\author{
Bojana Kisic, ${ }^{1}$ Dijana Miric, ${ }^{1}$ Ilija Dragojevic, ${ }^{1}$ \\ Julijana Rasic, ${ }^{2}$ and Ljiljana Popovic ${ }^{3}$ \\ ${ }^{1}$ Institute of Biochemistry, Medical Faculty Pristina, Kosovska Mitrovica 38220, Serbia \\ ${ }^{2}$ Institute of Pharmacology, Medical Faculty Pristina, Kosovska Mitrovica 38220, Serbia \\ ${ }^{3}$ Institute of Pathophysiology, Medical Faculty Pristina, Kosovska Mitrovica 38220, Serbia \\ Correspondence should be addressed to Bojana Kisic; bojanabk2002@yahoo.com
}

Received 11 February 2016; Accepted 14 March 2016

Academic Editor: Alexandra Scholze

Copyright (C) 2016 Bojana Kisic et al. This is an open access article distributed under the Creative Commons Attribution License, which permits unrestricted use, distribution, and reproduction in any medium, provided the original work is properly cited.

\begin{abstract}
Chronic kidney disease (CKD) is a worldwide public health problem. Patients with CKD have a number of disorders in the organism, and the presence of oxidative stress and systemic inflammation in these patients is the subject of numerous studies. Chronic inflammation joined with oxidative stress contributes to the development of numerous complications: accelerated atherosclerosis process and cardiovascular disease, emergence of Type 2 diabetes mellitus, development of malnutrition, anaemia, hyperparathyroidism, and so forth, affecting the prognosis and quality of life of patients with CKD. In this review we presented the potential role of the myeloperoxidase enzyme in the production of reactive/chlorinating intermediates and their role in oxidative damage to biomolecules in the body of patients with chronic kidney disease and end-stage renal disease. In addition, we discussed the role of modified lipoprotein particles under the influence of prooxidant MPO intermediates in the development of endothelial changes and cardiovascular complications in renal failure.
\end{abstract}

\section{Introduction}

Chronic kidney disease (CKD) is a widespread health problem. CKD progresses irreversibly and may lead to end-stage renal disease (ESRD). CKD and ESRD are linked to an increased risk of mortality, cardiovascular complications and comorbidities, and high costs for the treatment of renal failure with dialysis or transplantation. Chronic kidney disease is characterized by the accumulation and/or deficit of various substances in the human body and metabolic disorder caused by the primary disease and therapeutic procedure. Patients with CKD are exposed to increased risk of developing oxidative stress due to metabolic disorders, immune deficiency, and persistent inflammation [1]. Myeloperoxidase (MPO, EC 1.11.1.7) is a heme-containing enzyme found in mammalian neutrophils, where it catalyzes the hydrogen peroxide mediated peroxidation of halide ions and the pseudohalide thiocyanate. Products of these reactions and their secondary metabolites are responsible for killing phagocytized bacteria and viruses [2]. Myeloperoxidase is a $150-165-\mathrm{kDa}$ molecule synthesized during myeloid differentiation that constitutes the major component of the neutrophil azurophilic granules. The enzyme is a homodimer comprising of a pair of light and heavy chains derived from a single gene product with its subunits joined by a single disulfide bridge [3]. Upon phagocyte activation in peripheral blood, tissues, or fluids, MPO is released from leukocytes into both the phagolysosomal compartment and the extracellular milieu [4]. MPO and MPOderived oxidants may participate as mediators of oxidative modification of biomolecules/tissues and contribute to the development of comorbidities and complications in patient with CKD.

\section{Oxidative Stress and Inflammation in Chronic Kidney Disease}

Previous studies have shown that patients with chronic kidney disease (CKD) are exposed to a number of prooxidants 
that influence the occurrence of complications and poor outcomes. When increased production and/or inadequate removal of reactive oxygen species (ROS) overwhelm the redox homeostasis of the body, oxidative stress develops. The presence of toxin with prooxidant properties, malnutrition, systemic inflammation, weakened antioxidant defense system, drugs, and side effects of dialysis contribute to the disruption of redox homeostasis and development of oxidative stress in patients with CKD [5]. Reactive oxygen species play an important role in pathogenesis and progression of chronic kidney disease, being responsible for many complications that accompany this pathological condition, such as changes in the vasculature, endothelial dysfunction, atherosclerosis, and cardiovascular disease [6]. Results of numerous studies indicate an increase in concentration of markers of oxidative damage to biomolecules in plasma of patients with CKD. Among the most frequently studied are markers of oxidative damage to proteins (3-nitrotyrosine, carboxymethyllysine, and advanced oxidation protein products), lipids (malondialdehyde, 4-hydroxynonenal, lipohydroperoxides, oxycholesterols, F2-isoprostanes, and oxidized-LDL), and nucleic acids (DNA oxidation and RNA oxidation) [7].

Although dialysis provides a certain quality of life in patients with end-stage renal disease, long-term dialysis therapy causes many complications, probably due to imbalance between generation and elimination of reactive oxygen species. Materials of the hemodialysis membrane may affect both the formation of reactive species by activating polymorphonuclear cells and monocytes and the weakening of antioxidant capacity of the body due to the loss of hydrophilic antioxidants during dialysis, the consumption of liposoluble antioxidants, changes in the lipid composition of biological fluids, and/or deficit of coenzyme and antioxidant enzyme dysfunction [8].

Persistent inflammation is highly prevalent in CKD as a result of numerous disorders [9]. Although the true reasons are not fully elucidated, the immune system disorders in CKD include immunodeficiency and activation of immunocompetent cells, T lymphocytes, and monocytes and the production of proinflammatory cytokines by activated monocytes (TNF$\alpha$, IL-1 $\beta$, IL- 6 , etc.) as well as the production of chlorinated oxidants (such as $\mathrm{HOCl}$ ) by activated neutrophils. In the Chronic Renal Insufficiency Cohort (CRIC) study [10], the inverse correlation between glomerular filtration rate (GFR) and biomarkers of inflammation (IL-1 $\beta$, IL-1 receptor antagonist, IL-6, TNF- $\alpha$, C-reactive protein, and fibrinogen) was demonstrated as well as the direct correlation between kidney function and albuminuria. It was also found that the higher concentrations of acute phase reactants (C-reactive protein, CRP) and proinflammatory cytokines (especially IL6) strongly predicted cardiovascular morbidity and mortality in patients with CKD [11].

Hemodialysis (HD), as one of the therapeutic procedures performed on patients with renal failure, is believed to contribute to chronic inflammation due to exposure of blood to bioincompatible system causing activation of circulating phagocytes [12]. Hemobioincompatibility depends on the nature of the dialysis membrane and the possible endotoxin contamination of dialysate [8]. Activated neutrophils and monocytes produce reactive intermediate compounds that contribute to oxidative imbalance and development of proinflammatory oxidative stress responses where myeloperoxidase plays an important role. The increase in neutrophil degranulation products, myeloperoxidase and elastase, in HD patients was confirmed as compared to healthy people [13]. Production of oxidants starts upon activation of neutrophils, monocytes, and macrophages by proinflammatory mediators (IL-1 $\beta$, IL-6, and TNF- $\alpha$ ) when the activity of the NADPH oxidase (NOX-2) enzyme is enhanced. NADPH oxidase catalyzes the one-electron reduction of molecular $\mathrm{O}_{2}$ and the formation of superoxide anion radical $\left(\mathrm{O}_{2}{ }^{-}\right)$, where NADPH serves as electron donor [14]. When phagocytic cell is activated, NADPH oxidase accumulates in vesicles which bind to the cell membrane, and superoxide anion radical $\left(\mathrm{O}_{2}{ }^{--}\right)$is released in extracellular space or phagocytic vesicle.

\section{Myeloperoxidase as a Source of Oxidants in CKD}

Myeloperoxidase is a heme-containing enzyme, involved in oxygen-dependent mechanisms of microbicidal activity of professional phagocytes. MPO is most abundantly present in azurophilic granules of neutrophils, followed by monocytes and some macrophage subpopulations including resident tissue macrophages such as peritoneal macrophages, microglia, and Kupffer cells [15]. MPO catalyzes the reaction of hydrogen peroxide $\left(\mathrm{H}_{2} \mathrm{O}_{2}\right)$, formed by dismutation of $\mathrm{O}_{2}{ }^{--}$, and halide and pseudohalide ions $\left(\mathrm{Cl}^{-}, \mathrm{Br}^{-}\right.$, and $\left.\mathrm{SCN}^{-}\right)$ to the corresponding hypohalous acids (hypochlorous acid, $\mathrm{HOCl}$; hypobromous acid, $\mathrm{HOBr}$; and hypothiocyanous acid, HOSCN) [16] and formation of other reactive intermediates such as reactive nitrogen species and tyrosyl radicals (Figure 1). Oxidants produced by MPO are microbicidal factors and play an important role in innate immune response defending the body against bacteria, parasites, viruses, and other agents [17]. However, $\mathrm{HOCl}$ and other MPO-derived oxidants participate as mediators of oxidative damage to biomolecules, including proteins, nucleic acids, lipids, and carbohydrates, thus damaging the host tissue. In that way they may initiate and contribute to the development of atherosclerosis, endothelial dysfunction, cardiovascular, and other complications in patients with CKD [12]. $\mathrm{HOCl}$ can induce dysfunction of endothelial cells and affect endothelial function by decreasing the adhesiveness of extracellular matrix proteins for endothelial cells [18] and by converting matrix metalloproteinases into their active form, thus destabilizing the vascular and tissue environment surrounding endothelial cells [19].

Neutrophils are thought to be the main mediators of vascular inflammation [20]. Given that extracellular MPO is capable of catalyzing peroxidation of lipoproteins and other molecules in the blood, it is considered to be the main driving force for enzymatic lipid peroxidation in vivo. Lipid peroxidation of plasma lipoproteins possibly contributes to the development of atherosclerosis, commonly present in CKD patients. Due to the extremely cationic character, MPO can easily bind to the negatively charged structures, that is, bacteria, endothelial cells, cytokeratin-1, polyanionic matrix 


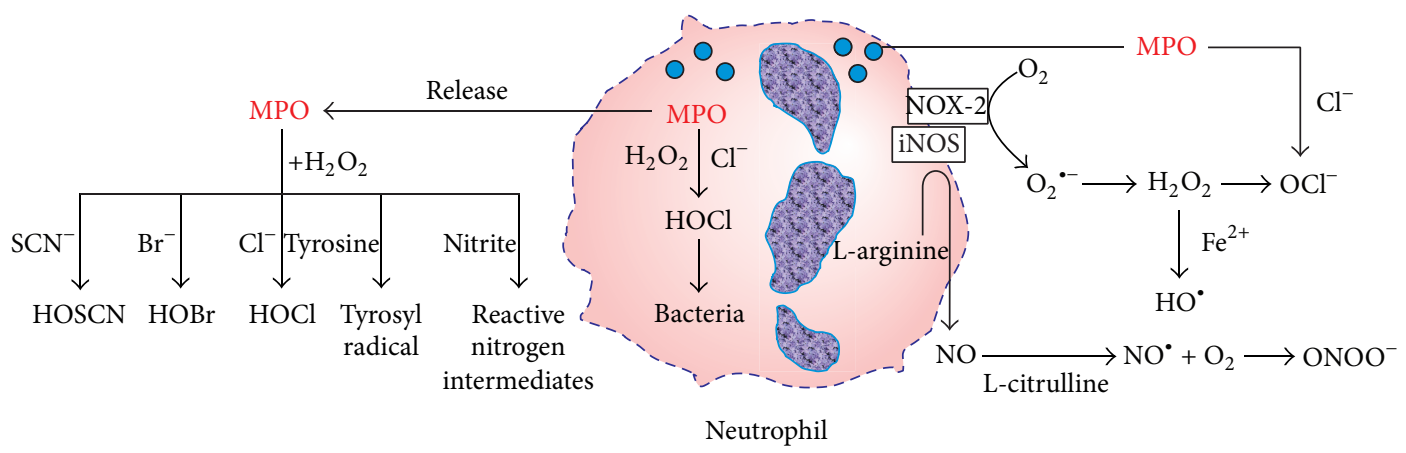

FIGURE 1: Reactive intermediates species formed by myeloperoxidase (modified from [17]).

glycosaminoglycans, apolipoproteins apoA-1 and apoB-100, ceruloplasmin, $\alpha 1$-antitrypsin, and albumin [21]. Hypochlorous acid formed through MPO-catalyzed oxidation of chlorine ions is a very strong oxidizing agent; that is, it is a strong oxidant with chlorinating properties [17]. Although $\mathrm{HOCl}$ is synthesized predominantly at the site of inflammation within phagocytic vacuole, the surrounding tissues can also suffer the adverse effects when $\mathrm{HOCl}$ leaks from incompletely closed phagocytic vacuole.

\section{MPO-Derived Oxidants and Protein Damage in CKD}

Under in vivo and in vitro conditions, $\mathrm{HOCl}$ can react with many biomolecules, with molecules that contain thiols, nitrogen compounds, or unsaturated double carbon bonds. In reaction with protein amino acid residues $\mathrm{HOCl}$ forms unstable mono- and dichloramines, which rapidly decompose forming aldehydes. The reaction of the $\mathrm{HOCl}$ with the tyrosine residues of proteins gives rise to 3,5-dichlorotyrosine (3,5-dichloro-Tyr) and 3-chlorotyrosine (3-chloroTyr) [22]. Glutathione sulphonamide is a specific biomarker of $\mathrm{MPO} / \mathrm{HOCl}$ production in vivo, as a product of $\mathrm{HOCl}-$ mediated glutathione oxidation [23]. Reactions of hypohalous acids with proteins contribute to protein unfolding and enzyme inactivation. Products of protein oxidation formed during the activity of phagocytes are considered as specific markers of MPO activity, and their increase in plasma indicates enhanced oxidative stress in CKD patients [13].

Albumin is the most abundant and the most important blood plasma protein. In physiological conditions, serum albumin constitutes about $50-60 \%$ of total plasma proteins. Human serum albumin is $\mathrm{Mr} 65 \mathrm{kDa}$ protein, composed of 585 amino acid residues and one free thiol (Cys 34) [24]. Albumin and low-molecular antioxidants of the blood plasma protect extracellular fluids from the oxidants generated by phagocytes. Also, albumin and plasma proteins, transferrin and ceruloplasmin, which bind transition metals and prevent them from participating as catalysts in reactions that lead to production of reactive species, are the main antioxidants in the extracellular environment. Albumin is a powerful scavenger of oxidants in human plasma that has the ability to inhibit hydroxyl radicals, peroxyl radicals, and $\mathrm{HOCl}$. Also, plasma proteins are very sensitive to the effects of reactive species and permanent exposure of albumin to oxidative stress causes its conformational and functional changes, thus affecting its biological properties. Sulfhydryl group ( $-\mathrm{SH}$ ) of albumin is very sensitive to the influence of $\mathrm{HOCl}$ and chloramine. Modified albumin irreversibly losses its biological properties. In patients undergoing maintenance HD the uremic milieu and therapeutic procedure itself, namely, dialysis, contribute to modification of albumin. Structural modifications of serum albumin affect its function in terms of the loss of ligand-binding capacity and other antioxidant properties.

Hypoalbuminemia present in patients with CKD is probably the result of combined effect of inflammation and inadequate protein and calorie intake. Inflammation reduces albumin concentration through cytokine production, reduces protein intake, and increases endogenous albumin catabolism by inducing anorexia. Since the serum albumin is an important antioxidant in the blood and extracellular environment, hypoalbuminemia contributes to the occurrence and development of oxidative stress in patients with CKD. In the human body albumin serves as a reservoir for $\mathrm{NO}$, so that hypoalbuminemia also reduces the bioavailability of NO and the endothelium-dependent vasodilatation. Some studies have shown that hypoalbuminemia in patients undergoing hemodialysis is unfavourable prognostic factor associated with the development of coronary heart disease [25] and also that the level of albumin in plasma has a significant effect on the survival in hemodialysis patients [26].

Advanced oxidation protein products (AOPP) were first proven biochemically and immunochemically in studies of Witko-Sarsat et al. [27] in plasma of hemodialysis patients. From chemical perspective, AOPP consist of various molecular aggregates of oxidatively modified proteins and are present as low-molecular weight AOPP (molecular weight of about $60 \mathrm{kDa}$ ) and high molecular weight AOPP (molecular weight of about $600 \mathrm{kDa}$ ). The AOPP are dominated by 3-chlorotyrosine, dityrosine, and 3-nitrotyrosine modifications, which confirm the assumption that under in vivo conditions AOPP are generated by the action of hypochlorous acid and chloramine produced by MPO. This assumption 
is confirmed by the evidence that the level of AOPP in plasma correlates with markers of monocyte activation, such as neopterin and TNF- $\alpha$ [28]. Biochemical analyses showed that the largest part of the serum AOPP consists of oxidized albumin, and its modification depends on MPO chlorination activity [29]. Polypeptide chain and its side groups are subject to oxidative changes. Also, in patients with CKD, uremic toxins contribute to production of AOPP because they stimulate monocyte-driven inflammatory process [28].

Chronically elevated blood urea, with the accumulation of toxic waste products inherent to a decline in glomerular filtration rate, has been proposed to contribute to the enhanced cardiovascular risk associated with CKD. High levels of urea in CKD facilitate posttranslational modification of proteins through a protein carbamylation [30]. Carbamylation of proteins results from nonenzymatic chemical modification by isocyanic acid derived from urea and an alternative route, the MPO-catalyzed oxidation of thiocyanate. Clinical studies have shown that measurement of circulating levels of protein carbamylation predicts incident cardiovascular risks in patients with CKD and in patients with end-stage renal disease undergoing hemodialysis [31]. Carbamylated albumin appears to be proinflammatory, carbamylated proteins are highly enriched in atherosclerotic plaques, and carbamylated LDL may be a pathogenic ligand for foam cells [32]. Many studies show that the carbamylation of proteins catalyzed by the leukocyte MPO is a dominant mechanism for carbamylation within human atherosclerotic lesions [33]. Urea, which is present in human body as a waste product of protein catabolism, slowly decomposes spontaneously in aqueous solutions forming cyanic acid (and its conjugate base, cyanate). Cyanic acid (HOCN) is in equilibrium with its reactive form, isocyanic acid (HNCO). The plasma concentration of HNCO in healthy individuals is estimated to be $\sim 50 \mathrm{nmol} / \mathrm{L}$ but can reach $150 \mathrm{nmol}$ in patients with CKD [31]. The active form of cyanate, isocyanic acid, is capable of interacting with $\mathrm{NH}_{2}$ groups of proteins and especially with the $\varepsilon-\mathrm{NH}_{2}$ groups of lysine residues, generating homocitrulline ( $\varepsilon$-N-C-lysine) [4]. The cyanate may also be generated via enzyme catalyzed oxidation of the pseudohalide thiocyanate $\left(\mathrm{SCN}^{-}\right)$by MPO [31]. The $\mathrm{SCN}^{-}$is a favoured substrate for MPO, with estimates that up to $50 \%$ of the $\mathrm{H}_{2} \mathrm{O}_{2}$ consumed by MPO oxidizes $\mathrm{SCN}^{-}$ under physiological conditions. Increasing evidence shows that protein carbamylation results in functional impairment of lipoproteins including high-density (HDL) and lowdensity lipoproteins (LDL) [30]. Although the mechanisms responsible for the formation of dysfunctional HDL are not completely characterized, there is strong evidence to support a role for MPO-derived chlorinating and nitrating oxidants [34].

\section{MPO as a Modulator of Immune Response in CKD}

Chloramines formed by the action of $\mathrm{HOCl}$ are toxic and inactivate the natural antiproteinase, such as $\alpha 1$-antitrypsin, while in vitro $\mathrm{HOCl}$ causes fragmentation of immunoglobulin molecules, suggesting that oxidants generated by MPO can compromise the adaptive host immune response. In addition, activated neutrophils secrete numerous proteases, including cathepsin G. This serine protease plays a role in the release of cytokines, activation of receptors, and degradation of tissue proteins.

There is evidence that MPO also functions as an autocrine modulator of neutrophil function, by recruiting previously unstimulated neutrophils. Extracellular MPO adheres to cell membrane via CD11b/CD18 neutrophil integrins. It is followed by tyrosine phosphorylation and activation of $\mathrm{p} 38$ MAP kinase, translocation of nuclear factor $\kappa \mathrm{B}$, and elevated expression of fibronectin and CD11 integrin as well as increased production of superoxide anion radical $\left(\mathrm{O}_{2}{ }^{--}\right)$due to activation of membrane NADPH oxidase [35]. This kind of recruitment of new neutrophils increases the production of oxidants in the vascular compartment during inflammation. In addition, MPO can adhere to leukocytes via binding to $\mathrm{CD} 11 \mathrm{~B} / \mathrm{CD} 18$ which may contribute to the proinflammatory effect of MPO through the accumulation of leukocytes at the inflammation site [15]. There are also reports that even enzymatically inactive MPO may affect the activation of endothelial cells and the production of cytokines, IL- 6 and IL-8 [36], which is another proinflammatory effect of MPO.

\section{MPO Genetic Polymorphism and CKD}

It is well known that a single-nucleotide $\mathrm{G}$ to A polymorphism can occur in the -463 promoter region of MPO gene, located on chromosome 17 , associated with altered MPO expression. The presence of G-allele rather than Aallele increases the expression of MPO, which can be the reason of higher enzyme activity in atherosclerosis as well as several malignant, degenerative, and inflammatory diseases. However, the results of clinical studies regarding MPO functional polymorphism and risk factors of kidney damage seem to be controversial. The study of Cayley Jr. et al. [37] has shown that predominantly GG genotype was associated with higher levels of circulating MPO and increased risk of cardiovascular disease. GG genotype was also associated with increased incidence of cardiovascular disease in predialysis CKD patients [38]. Moreover, peripheral neutrophils of GG genotype showed increased production of ROS [39]. Still, the same study reported no differences in MPO genotypes frequencies between healthy controls and CKD patients, with exception in those with hypertensive nephrosclerosis who showed slightly increased frequency of GG genotype [39]. On the other hand, Liu et al. [40] have shown that the presence of A-allele was associated with almost doubled risk of hypertension, which is one of the most important causative factors leading to $\mathrm{CKD}$, while among African American patients with systemic lupus erythematosus the low expression of A-allele was associated with a higher risk of lupus nephritis development [41].

\section{MPO and Renal Replacement Therapy in CKD}

In conservatively treated clinically stable CKD patients, serum MPO was reported to be normal [42] and even 
decreasing with aggravation of kidney function [43]. In patients who were already on renal replacement therapy, either HD or continuous ambulatory peritoneal dialysis (CAPD), basal serum MPO activity or concentration was reported to increase, with the highest values found in CAPD $[28,44,45]$. Moreover, in patients undergoing maintenance HD treatment the higher basal serum MPO was associated with inflammation, advanced atherosclerosis, and poorer prognosis $[44,46]$. On the other hand, basal serum MPO activity in HD patients was in several studies within the range of healthy controls $[47,48]$, except in cases with arteriovenous fistula thrombosis [49]. These discrepant results probably reflect many confounding factors, such as the duration of HD treatment, use of different dialysis membranes/reuse of a dialyzer, different proportion of underlying causes of CKD, MPO genetic polymorphisms, comorbidities, vitamin, mineral, and antioxidant supplementation, and lipid lowering drugs. Still, MPO-deficiency was recently demonstrated to retard the progression of CKD in 5/6 nephrectomized mice [50], thereby confirming the role of MPO in kidney damage.

The other question is how extracellular MPO is influenced by dialysis procedure itself, and if so, what would be the consequences? Maintenance HD is currently the most preferable renal replacement modality in CKD patients, followed by CAPD. These procedures certainly bring some relief by removing low-molecular weight toxins and oxidation products from the body and maintaining water and ionic balance. Still, the contact of the blood with bioincompatible dialysis membrane or fluid impurity may provoke degranulation of neutrophils and release of MPO, elastase, and other bioactive molecules into vascular compartment. These compounds may further enhance the production of ROS accompanied by formation of more advanced oxidation products and toxic mediators and contribute to proinflammatory and prothrombotic environment, leading to vascular dysfunction [8]. Maintenance HD treatment is usually performed thrice a week, and MPO repetitively released from neutrophils into vascular compartment is highly likely to play an important role in oxidative stress and complications in CKD [28].

Previous studies have shown that even a single HD session would result in severalfold increase in serum MPO activity or concentration $[45,48,51]$, but regardless of the magnitude of increment, MPO would usually return to normal well before initiation of the next HD session (Author's unpublished data). Aside from membrane compatibility, there could be at least two other causes for this increment. Using biocompatible membranes and ultrapure dialysis fluid Rutgers and coworkers [52] examined the change in peripheral blood neutrophil MPO activity after 4 hours of $\mathrm{HD}$ in $54 \mathrm{CKD}$ patients and 12 healthy volunteers. The results indicated that, unlike in healthy subjects, a single HD treatment was capable of significantly increasing MPO in neutrophils of CKD patients, not only due to MPO genetic polymorphism, but also due to dialysis procedure itself [52]. The other important, although underestimated, cause of intradialytic increase in serum MPO could be endotheliumbound enzyme, released during HD into circulating blood when patients are anticoagulated with heparin, instead of trisodium citrate [53]. That increase rapidly occurred within the first 10-15 minutes, reaching severalfold higher MPO levels than the baseline, decreased during HD session, although still being elevated after completion of procedure [51, 53].

It is however unclear what the consequences of this tidal change of circulating MPO in maintenance HD patients would be. As a strongly cationic protein MPO may reversibly attach to negatively charged groups expressed on endothelial surface glycosaminoglycan-rich receptors and become transiently removed from the circulating blood. Both unfractionated and low-molecular weight heparins compete with MPO for receptors, and bolus injection of these anticoagulants induces the release of MPO into the blood stream [53]. The detachment of MPO from endothelium could be beneficial for patient, since endothelium-bound enzyme may transverse into intima/media where it can act as a prooxidant and proinflammatory agent implicated in atheromatous plaque development. On the other hand, once released into the bloodstream MPO may disseminate to remote tissues thereby accelerating generalized atherosclerosis.

\section{MPO, Lipid Disorders, and Lipid Peroxidation in CKD}

Cardiovascular diseases and complications are the leading cause of morbidity and mortality in patients with CKD, primarily due to atherosclerotic disease [54]. Lipid oxidation/chlorination by the $\mathrm{MPO} / \mathrm{HOCl}$ system could be important player in initiation and progression of atherosclerotic changes [55]. The oxidation of low-density lipoproteins (LDL) by $\mathrm{HOCl}$ generated in MPO-catalyzed reaction is thought to be a proatherogenic event which precedes the formation of foam cells, a hallmark of atherosclerotic plaque development [56]. Oxidatively modified lipoproteins have reduced functional ability for reverse cholesterol transport, and thus formed lipid peroxides may influence activation, adhesion, infiltration, and differentiation of monocytes as well as the production of MPO and its oxidizing products.

Dyslipidemia, that is, impaired concentration and structure, as well as the metabolism of lipoproteins, is an important factor that increases the risk of coronary heart disease in patients with CKD [57]. The most common blood lipid disorders in CKD are hypertriglyceridemia and increased concentration of atherogenic triglyceriderich lipoprotein species, such as very low-density lipoproteins (VLDL) and intermediate-density lipoproteins (IDL), while the concentration of high-density lipoproteins (HDL) is decreased [58]. Decreased activity of lipoprotein lipase (LPL) and slow catabolism of triglyceride-rich lipoprotein particles contribute to the development of dyslipidemia in patients with renal insufficiency. LPL is synthesized mainly in adipocytes and myocytes, but, like MPO, when released into the circulation it becomes attached to endothelial cell surface via heparan-sulfate proteoglycans. LPL is implicated mainly in metabolism of chylomicrons and VLDL, while the hepatic lipase mainly metabolizes IDL and LDL. It is believed that endothelial lipase enzyme is responsible for the lipolytic catabolism of HDL in maintenance HD patients [59]. Numerous factors act as inhibitors of lipolytic enzymes in uremic patients: systemic inflammation, oxidative stress, 
oxidative modification and carbamylation of apolipoproteins, downregulation of gene encoding lipoprotein lipase, and many others [60]. Apolipoprotein C-III is a potent inhibitor, whereas the apolipoprotein C-II is an activator of lipoprotein lipase, and for the normal functioning lipoprotein lipase requires the presence of apoC-II, as a cofactor [61]. Increased permeability of the glomerular membrane leads to the loss of apoC-II, a lipoprotein lipase activator. A decrease of apolipoprotein C-II/C-III ratio due to a disproportionate increase of apolipoprotein C-III in plasma is a possible cause of lipoprotein lipase inactivation in patients with renal insufficiency $[62,63]$. Probably a number of mechanisms are responsible for the increase in the concentration of apolipoprotein C-III in the plasma of these patients. Impaired renal function contributes to the above-mentioned, because the kidneys are involved in the elimination of apoC-III from the plasma. The results of some tests [64] pointed out that the mediators of inflammation in the blood of patients with CKD increase apoC-III gene expression and that inflammation with an increased concentration of apoC-III delays VLDL apoB-100 catabolism and its subclasses [63].

The proportion of LDL, which are considered to have a distinct atherogenic potential, was significantly increased in $\mathrm{CKD}$. There were also qualitative changes in LDL particles that involve oxidative modification of LDL and decrease in HDL protection against LDL oxidation. Oxidized-LDL plays an important role in the development and progression of atherosclerotic changes [65]. In addition to inducing endothelial dysfunction, oxidized-LDL particles exhibit proinflammatory activity including chemotactic effects and expression of macrophage colony-stimulating factors, affecting the adhesion of molecules and monocyte activation [66]. MPO and MPO-derived oxidant products contribute to atherogenesis in patients with CKD [67]. The MPO-derived oxidant products induce oxidation of both protein and lipid components of LDL, resulting in the formation of a modified particle, so-called MPO-dependent oxidized-LDL [68]. Oxidative modification of LDL is not limited to the intima of blood vessel but also occurs within the blood plasma. In the state of persistent inflammation when other antioxidants are deprived or at a highly oxidized state, such as in CKD, increased presence of extracellular MPO could be the major initiator of lipoprotein oxidative modifications. In lipoproteins of blood plasma, as a reflection of the slow oxidation, a small amount of autooxidized fatty acids is always present in the form of relatively stable organic hydroperoxides. In the presence of $\mathrm{HOCl}$, these hydroperoxides are converted into peroxyl radicals and singlet oxygen is produced [69]. The study by Delporte et al. [68] confirmed the presence of higher concentrations of MPO-dependent oxidized-LDL (Mox-LDL), as a potential marker of plasma MPO activity in patients undergoing HD treatment.

Beside apoB-100 modifications, MPO-derived oxidants also induce oxidative changes in lipid components of LDL, causing peroxidation of unsaturated fatty acids in phospholipids and fatty acids esterified with glycerol and cholesterol within LDL particles [70]. Lysolecithin is generated in LDL particle as a result of the lipid peroxidation, and the concentration of esterified cholesterol is reduced, a number of lipid-derived oxidation products are formed, including lipid hydroperoxides, and specific products of linoleate and arachidonate oxidation are formed, including 9-hydroxy10,12-octadecadienoic acid (9-HODE) and F2-isoprostanes $[70,71]$.

Several experimental and clinical studies confirmed the accumulation of oxidized low-density lipoproteins (oxLDL) in circulation and renal interstitium in CKD [72]. In vitro studies indicate that oxLDL stimulate mesangial cells to secrete chemoattractant chemokines that attract monocytes [73]. Animal studies have shown that chronic exposure to oxLDL stimulates collagen synthesis and activation of proinflammatory pathways in kidneys [72].

In addition to the transport of excess cholesterol to the liver for further metabolizing, HDL particles play a role as endogenous inhibitors of inflammation, platelet aggregation, and LDL oxidation. A key cardioprotective feature of HDL particles is their participation in reverse cholesterol transport (using apolipoprotein A-I, an integral part of $\mathrm{HDL}$ ), which removes excess cholesterol from the cells (including macrophages in the artery wall) and transports it to the liver [74]. In CKD, the level of HDL-cholesterol in the blood is lower compared to those with normal renal function, and there are structural modifications and impairment of the HDL particles function. Evidences suggest that MPO is the main enzyme involved in chlorination and nitration of lipoproteins [75]. Analysing chlorinated apoA-I, it has been found that the loss of its activity was the result of oxidation of several methionine residues and chlorination of a single tyrosine residue Tyr192 [76]. Moreover, the reactive lipid peroxidation products such as malondialdehyde (MDA) can modify and cross-link specific residues in apo-AI, causing functional changes in HDL [77] and contributing to atherogenesis due to impaired cholesterol efflux from macrophages [76].

Reverse cholesterol transport is exacerbated in patients with CKD also due to low activity of lecithin cholesterol acyltransferase (LCAT) and paraoxonase. Reduced activity of LCAT enzyme [78] and at the same time increased activity of cholesteryl ester transfer protein [79] may further decrease HDL in patients with impaired renal function. In CKD patients HDL particles have weakened antioxidative and antiinflammatory properties, possibly due to reduced activity of HDL-associated enzymes, such as paraoxonase [80]. The amount of oxidatively modified HDL particles (oxHDL) is associated with protein-energy wasting in patients undergoing hemodialysis [81]. The loss of serum proteins in dialysis effluent can stimulate the synthesis of albumin and other proteins of the liver, including also a lipoprotein-enriched cholesterol.

\section{Reactive Nitrogen Species in Chronic Kidney Disease}

In the state of inflammation in the body of patients with CKD, oxidative stress is followed by the effects of nitrogen stress. Nitric oxide (NO*) has a significant role in the regulation of vascular tone and arterial tension, prevents platelet aggregation and adhesion of leukocytes to the wall of the blood vessel (by downregulation of endothelial adhesion molecules), 
prevents the proliferation of smooth-muscle cells of blood vessels, but also exerts adverse effects due to increase in concentration or changes in the oxidation state [82]. NO synthesis is carried out by the influence of the enzymes nitric oxide synthases (NOS), which in the presence of molecular oxygen reduce the substrate $\mathrm{L}$-arginine to $\mathrm{L}$-citrulline. However, the bioavailability of $\mathrm{NO}$ in the body depends not only on its synthesis, but also on its degradation mediated by ROS, particularly superoxide anion $\left(\mathrm{O}_{2}{ }^{--}\right)$[83]. Nitric oxide rapidly reacts with superoxide radical $\left(\mathrm{O}_{2}{ }^{\cdot-}\right)$ forming the highly reactive peroxynitrite $\left(\mathrm{ONOO}^{-}\right)$, which is a reactive compound of nonradical type and can directly start the process of lipid peroxidation, or indirectly, by reacting with reduced glutathione (GSH), an important factor for antioxidant defense. Peroxynitrite and its protonated form (peroxynitric acid, $\mathrm{ONOOH}$ ) are strong oxidants; therefore, peroxynitrite leads to nitrosative and oxidative modifications to proteins, nucleic acids, and lipids. The resultant oxidation products such as 3-nitrotyrosine and lipid hydroperoxides accumulate in the fibrotic kidneys as well as in the plasma of patients with chronic kidney disease, suggesting that oxidative stress intensifies during the progression of kidney disease [84]. MPO can catalyze the oxidation of $\mathrm{NO}$ and/or its metabolite $\mathrm{NO}_{2}{ }^{-}$to the more reactive nitrogen species and thus contribute to a thiol nitrosation and protein tyrosine nitration [85].

Researching endothelial dysfunction in patients undergoing dialysis, which is prior to structural changes in the vascular tree and the consequent clinical changes in blood vessels, Stenvinkel et al. [86] confirmed that endothelial dysfunction is associated with reduced NO bioavailability and increased concentration of its endogenous inhibitor: asymmetric dimethylarginine (ADMA) concentration in serum. Namely, ADMA is synthesized when L-arginine incorporated into proteins is methylated by the enzyme protein-arginine methyltransferase during posttranslational protein modification [87]. When methylated proteins are hydroxylated during transport of proteins, ADMA residues are released. Due to the very similar structure to L-arginine, ADMA may act as an endogenous competitive inhibitor of NO synthase [88]. In patients undergoing hemodialysis, elevated level of ADMA is associated with a higher risk of cardiovascular disease and mortality [89]. Also, the increased concentration of ADMA is associated with increased morbidity, mortality, and graft failure in kidney transplant recipients [90].

Along with the formation of ADMA, its inactive stereoisomer symmetrical dimethylarginine (SDMA) is created. SDMA does not directly inhibit NO synthase but is a competitor of L-arginine transport and impairs L-arginine uptake from the loop of Henle, which limits the availability of Larginine to NO synthase [91]. During the study by Schepers et al. [92], it was proven that SDMA has a proinflammatory effect through stimulation of generating ROS in monocytes, which can be a driver of vascular damage.

\section{Conclusion}

Examination of the effect of oxidative stress on the occurrence and development of CKD is important for assessing the degree of damage, possibility of slowing further development of the disease, and delaying the development of complications and comorbidities. Activation of neutrophils, release of myeloperoxidase, and the consequent formation of reactive/chlorinating intermediate products contribute to persistent inflammation and oxidative damage to biomolecules in the body of patients with CKD. MPO-derived reactive species (e.g., hypochlorous acid) may influence cell function and contribute to tissue damage. Oxidatively modified lipids and proteins contribute to the initiation and propagation of atherosclerotic changes and the development of cardiovascular complications in CKD. Despite progress made in understanding the complex mechanisms of MPO and MPOderived reactive intermediates activities, further research in order to determine the exact role in the development of oxidative stress and inflammation in renal failure would be certainly justified.

\section{Competing Interests}

The authors declare that they have no competing interests.

\section{References}

[1] M. Zargari and O. Sedighi, "Influence of hemodialysis on lipid peroxidation, enzymatic and non-enzymatic antioxidant capacity in chronic renal failure patients," Nephro-Urology Monthly, vol. 7, no. 4, Article ID e28526, 2015.

[2] M. Zederbauer, W. Jantschko, K. Neugschwandtner et al., "Role of the covalent glutamic acid 242-heme linkage in the formation and reactivity of redox intermediates of human myeloperoxidase," Biochemistry, vol. 44, no. 17, pp. 6482-6491, 2005.

[3] Y. R. Tahboub, S. Galijasevic, M. P. Diamond, and H. M. AbuSoud, "Thiocyanate modulates the catalytic activity of mammalian peroxidases," The Journal of Biological Chemistry, vol. 280, no. 28, pp. 26129-26136, 2005.

[4] D. I. Pattison, M. J. Davies, and C. L. Hawkins, "Reactions and reactivity of myeloperoxidase-derived oxidants: differential biological effects of hypochlorous and hypothiocyanous acids," Free Radical Research, vol. 46, no. 8, pp. 975-995, 2012.

[5] D. M. Small, J. S. Coombes, N. Bennett, D. W. Johnson, and G. C. Gobe, "Oxidative stress, anti-oxidant therapies and chronic kidney disease," Nephrology, vol. 17, no. 4, pp. 311-321, 2012.

[6] D. E. Weiner and M. J. Sarnak, "A decade after the KDOQI CKD guidelines: impact on the cardiovascular disease-CKD paradigm," American Journal of Kidney Diseases, vol. 60, no. 5, pp. 710-712, 2012.

[7] P. S. Tucker, V. J. Dalbo, T. Han, and M. I. Kingsley, "Clinical and research markers of oxidative stress in chronic kidney disease," Biomarkers, vol. 18, no. 2, pp. 103-115, 2013.

[8] M. Morena, J.-P. Cristol, J.-Y. Bosc et al., "Convective and diffusive losses of vitamin $\mathrm{C}$ during haemodiafiltration session: a contributive factor to oxidative stress in haemodialysis patients," Nephrology Dialysis Transplantation, vol. 17, no. 3, pp. 422-427, 2002.

[9] G. A. Kaysen, "Biochemistry and biomarkers of inflamed patients: why look, what to assess," Clinical Journal of the American Society of Nephrology, vol. 4, supplement 1, pp. S56S63, 2009. 
[10] J. Gupta, N. Mitra, P. A. Kanetsky et al., "Association between albuminuria, kidney function, and inflammatory biomarker profile in CKD in CRIC," Clinical Journal of the American Society of Nephrology, vol. 7, no. 12, pp. 1938-1946, 2012.

[11] G. Tripepi, F. Mallamaci, and C. Zoccali, "Inflammation markers, adhesion molecules, and all-cause and cardiovascular mortality in patients with ESRD: searching for the best risk marker by multivariate modeling," Journal of the American Society of Nephrology, vol. 16, no. 3, supplement 1, pp. S83-S88, 2005.

[12] C. Libetta, V. Sepe, P. Esposito, F. Galli, and A. Dal Canton, "Oxidative stress and inflammation: implications in uremia and hemodialysis," Clinical Biochemistry, vol. 44, no. 14-15, pp. 11891198, 2011.

[13] J. Himmelfarb, M. E. McMenamin, G. Loseto, and J. W. Heinecke, "Myeloperoxidase-catalyzed 3-chlorotyrosine formation in dialysis patients," Free Radical Biology and Medicine, vol. 31, no. 10, pp. 1163-1169, 2001.

[14] D. C. Abrantes, T. M. C. Brunini, C. Matsuura et al., "Diminished nitric oxide generation from neutrophils suppresses platelet activation in chronic renal failure," Molecular and Cellular Biochemistry, vol. 401, no. 1-2, pp. 147-153, 2015.

[15] D. Odobasic, A. R. Kitching, and S. R. Holdsworth, "Neutrophil-mediated regulation of innate and adaptive immunity: the role of myeloperoxidase," Journal of Immunology Research, vol. 2016, Article ID 2349817, 11 pages, 2016.

[16] B. S. Rayner, D. T. Love, and C. L. Hawkins, "Comparative reactivity of myeloperoxidase-derived oxidants with mammalian cells," Free Radical Biology and Medicine, vol. 71, pp. 240-255, 2014.

[17] T. N. Mayadas, X. Cullere, and C. A. Lowell, "The multifaceted functions of neutrophils," Annual Review of Pathology: Mechanisms of Disease, vol. 9, pp. 181-218, 2014.

[18] M. D. Rees, L. Dang, T. Thai, D. M. Owen, E. Malle, and S. R. Thomas, "Targeted subendothelial matrix oxidation by myeloperoxidase triggers myosin II-dependent de-adhesion and alters signaling in endothelial cells," Free Radical Biology and Medicine, vol. 53, no. 12, pp. 2344-2356, 2012.

[19] X. Fu, S. Y. Kassim, W. C. Parks, and J. W. Heinecke, "Hypochlorous acid generated by myeloperoxidase modifies adjacent tryptophan and glycine residues in the catalytic domain of matrix metalloproteinase-7 (matrilysin). An oxidative mechanism for restraining proteolytic activity during inflammation," Journal of Biological Chemistry, vol. 278, no. 31, pp. 28403-28409, 2003.

[20] M. Phillipson and P. Kubes, "The neutrophil in vascular inflammation,” Nature Medicine, vol. 17, no. 11, pp. 1381-1390, 2011.

[21] M. J. Davies, "Myeloperoxidase-derived oxidation: mechanisms of biological damage and its prevention," Journal of Clinical Biochemistry and Nutrition, vol. 48, no. 1, pp. 8-19, 2011.

[22] D. I. Pattison and M. J. Davies, "Absolute rate constants for the reaction of hypochlorous acid with protein side chains and peptide bonds," Chemical Research in Toxicology, vol. 14, no. 10, pp. 1453-1464, 2001.

[23] D. T. Harwood, A. J. Kettle, S. Brennan, and C. C. Winterbourn, "Simultaneous determination of reduced glutathione, glutathione disulphide and glutathione sulphonamide in cells and physiological fluids by isotope dilution liquid chromatographytandem mass spectrometry," Journal of Chromatography B: Analytical Technologies in the Biomedical and Life Sciences, vol. 877, no. 28, pp. 3393-3399, 2009.

[24] P.-S. Lim, Y.-M. Cheng, and S.-M. Yang, "Impairments of the biological properties of serum albumin in patients on haemodialysis," Nephrology, vol. 12, no. 1, pp. 18-24, 2007.
[25] T. Panaput, B. Thinkhamrop, S. Domrongkitchaiporn et al., "Dialysis dose and risk factors for death among ESRD patients treated with twice-weekly hemodialysis: a prospective cohort study," Blood Purification, vol. 38, no. 3-4, pp. 253-262, 2014.

[26] T. B. Pifer, K. P. McCullough, F. K. Port et al., "Mortality risk in hemodialysis patients and changes in nutritional indicators: DOPPS," Kidney International, vol. 62, no. 6, pp. 2238-2245, 2002.

[27] V. Witko-Sarsat, M. Friedlander, T. N. Khoa et al., "Advanced oxidation protein products as novel mediators of inflammation and monocyte activation in chronic renal failure," Journal of Immunology, vol. 161, no. 5, pp. 2524-2532, 1998.

[28] C. Capeillère-Blandin, V. Gausson, A. T. Nguyen, B. DescampsLatscha, T. Drüeke, and V. Witko-Sarsat, "Respective role of uraemic toxins and myeloperoxidase in the uraemic state," Nephrology Dialysis Transplantation, vol. 21, no. 6, pp. 1555$1563,2006$.

[29] C. Capeillère-Blandin, V. Gausson, B. Descamps-Latscha, and V. Witko-Sarsat, "Biochemical and spectrophotometric significance of advanced oxidized protein products," Biochimica et Biophysica Acta (BBA)-Molecular Basis of Disease, vol. 1689, no. 2, pp. 91-102, 2004.

[30] S. Kalim, S. A. Karumanchi, R. I. Thadhani, and A. H. Berg, "Protein carbamylation in kidney disease: pathogenesis and clinical implications," American Journal of Kidney Diseases, vol. 64, no. 5, pp. 793-803, 2014.

[31] F. H. Verbrugge, W. H. W. Tang, and S. L. Hazen, "Protein carbamylation and cardiovascular disease," Kidney International, vol. 88, no. 3, pp. 474-478, 2015.

[32] A. H. Berg, C. Drechsler, J. Wenger et al., "Carbamylation of serum albumin as a risk factor for mortality in patients with kidney failure," Science Translational Medicine, vol. 5, no. 175, Article ID 175ra29, 2013.

[33] Z. Wang, S. J. Nicholls, E. R. Rodriguez et al., "Protein carbamylation links inflammation, smoking, uremia and atherogenesis," Nature Medicine, vol. 13, no. 10, pp. 1176-1184, 2007.

[34] K. A. Hadfield, D. I. Pattison, B. E. Brown et al., "Myeloperoxidase-derived oxidants modify apolipoprotein A-I and generate dysfunctional high-density lipoproteins: comparison of hypothiocyanous acid (HOSCN) with hypochlorous acid ( $\mathrm{HOCl})$," Biochemical Journal, vol. 449, no. 2, pp. 531-542, 2013.

[35] D. Lau, H. Mollnau, J. P. Eiserich et al., "Myeloperoxidase mediates neutrophil activation by association with CD11b/CD18 integrins," Proceedings of the National Academy of Sciences of the United States of America, vol. 102, no. 2, pp. 431-436, 2005.

[36] D. L. Lefkowitz, E. Roberts, K. Grattendick et al., "The endothelium and cytokine secretion: the role of peroxidases as immunoregulators," Cellular Immunology, vol. 202, no. 1, pp. 23-30, 2000.

[37] W. E. Cayley Jr., F. W. Asselbergs, J.-W. C. Tervaert et al., "Prognostic value of myeloperoxidase in patients with chest pain," New England Journal of Medicine, vol. 350, no. 5, pp. 516-518, 2004.

[38] R. Pecoits-Filho, P. Stenvinkel, A. Marchlewska et al., "A functional variant of the myeloperoxidase gene is associated with cardiovascular disease in end-stage renal disease patients," Kidney International. Supplement, vol. 63, supplement 84, pp. S172-S176, 2003.

[39] K. Doi, E. Noiri, R. Maeda et al., "Functional polymorphism of the myeloperoxidase gene in hypertensive nephrosclerosis dialysis patients," Hypertension Research, vol. 30, no. 12, pp. 1193-1198, 2007. 
[40] Y.-C. Liu, C.-J. Chung, H.-S. Shiue et al., "Genetic polymorphisms of myeloperoxidase and their effect on hypertension," Blood Pressure, vol. 22, no. 5, pp. 282-289, 2013.

[41] H. Bouali, P. Nietert, T. M. Nowling et al., "Association of the G463A myeloperoxidase gene polymorphism with renal disease in African Americans with systemic lupus erythematosus," Journal of Rheumatology, vol. 34, no. 10, pp. 2028-2034, 2007.

[42] G. Caimi, C. Carollo, M. Montana, F. Vaccaro, and R. L. Presti, "Elastase, myeloperoxidase, nitric oxide metabolites and oxidative status in subjects with clinical stable chronic renal failure on conservative treatment," Clinical Hemorheology and Microcirculation, vol. 43, no. 3, pp. 253-258, 2009.

[43] M. A. Rao, U. Anand, and C. V. Anand, "Myeloperoxidase in chronic kidney disease," Indian Journal of Clinical Biochemistry, vol. 26, no. 1, pp. 28-31, 2011.

[44] K. Kalantar-Zadeh, M.-L. Brennan, and S. L. Hazen, "Serum myeloperoxidase and mortality in maintenance hemodialysis patients," American Journal of Kidney Diseases, vol. 48, no. 1, pp. 59-68, 2006.

[45] A. Al-Hweish, S. S. Sultan, K. Mogazi, and M. Y. Elsammak, "Plasma myeloperoxidase, NT-proBNP, and troponin-I in patients on CAPD compared with those on regular hemodialysis," Hemodialysis International, vol. 14, no. 3, pp. 308-315, 2010.

[46] K. C. Hsiao, J. P. Tsai, S. F. Yang et al., "MMP-2 serum concentrations predict mortality in hemodialysis patients: a 5 -year cohort study," Clinica Chimica Acta, vol. 452, pp. 161-166, 2016.

[47] H. Honda, M. Ueda, S. Kojima et al., "Assessment of myeloperoxidase and oxidative $\alpha_{1}$-antitrypsin in patients on hemodialysis," Clinical Journal of the American Society of Nephrology, vol. 4, pp. 142-151, 2009.

[48] D. Miric, B. Kisic, R. Stolic, B. Miric, R. Mitic, and S. JanicijevicHudomal, "The role of xanthine oxidase in hemodialysisinduced oxidative injury: relationship with nutritional status," Oxidative Medicine and Cellular Longevity, vol. 2013, Article ID 245253, 8 pages, 2013.

[49] R. V. Stolic, G. Z. Trajkovic, D. J. Miric et al., "Arteriovenous fistulas and digital hypoperfusion ischemic syndrome in patients on hemodialysis," World Journal of Nephrology, vol. 2, no. 2, pp. 26-30, 2013.

[50] A. Lehners, S. Lange, G. Niemann et al., "Myeloperoxidase deficiency ameliorates progression of chronic kidney disease in mice," American Journal of Physiology-Renal Physiology, vol. 307, no. 4, pp. F407-F417, 2014.

[51] J. Gozdzikiewicz, J. Borawski, E. Koc-Zorawska, and M. Mysliwiec, "Effects of enoxaparin on myeloperoxidase release during hemodialysis," Hemodialysis International, vol. 18, no. 4, pp. 819-824, 2014.

[52] A. Rutgers, P. Heeringa, J. P. Kooman, F. M. van der Sande, and J. W. C. Tervaert, "Peripheral blood myeloperoxidase activity increases during hemodialysis," Kidney International, vol. 64, article 760, 2003.

[53] J. Borawski, "Myeloperoxidase as a marker of hemodialysis biocompatibility and oxidative stress: the underestimated modifying effects of heparin," American Journal of Kidney Diseases, vol. 47, no. 1, pp. 37-41, 2006.

[54] A. S. Go, G. M. Chertow, D. Fan, C. E. McCulloch, and C.-Y. Hsu, "Chronic kidney disease and the risks of death, cardiovascular events, and hospitalization," The New England Journal of Medicine, vol. 351, no. 13, pp. 1296-1305, 2004.
[55] B. Shao, C. Tang, A. Sinha et al., "Humans with atherosclerosis have impaired ABCAl cholesterol efflux and enhanced highdensity lipoprotein oxidation by myeloperoxidase," Circulation Research, vol. 114, no. 11, pp. 1733-1742, 2014.

[56] A. Mahrooz, M. Zargari, O. Sedighi, H. Shaygani, and G. Gohari, "Increased oxidized-LDL levels and arylesterase activity/HDL ratio in ESRD patients treated with hemodialysis," Clinical and Investigative Medicine, vol. 35, no. 3, pp. E144-E151, 2012.

[57] W. F. Keane, J. E. Tomassini, and D. R. Neff, "Lipid abnormalities in patients with chronic kidney disease: implications for the pathophysiology of atherosclerosis," Journal of Atherosclerosis and Thrombosis, vol. 20, no. 2, pp. 123-133, 2013.

[58] S. S. Prichard, "Impact of dyslipidemia in end-stage renal disease," Journal of the American Society of Nephrology, vol. 14, no. 9, supplement 4, pp. S315-S320, 2003.

[59] V. Miksztowicz, M. G. McCoy, L. Schreier et al., "Endothelial lipase activity predicts high-density lipoprotein catabolism in hemodialysis: novel phospholipase assay in postheparin human plasma," Arteriosclerosis, Thrombosis, and Vascular Biology, vol. 32, no. 12, pp. 3033-3040, 2012.

[60] P. Barter, "Lipoprotein metabolism and CKD: overview," Clinical and Experimental Nephrology, vol. 18, no. 2, pp. 243-246, 2014.

[61] S. Y. Kim, S. M. Park, and S.-T. Lee, "Apolipoprotein C-II is a novel substrate for matrix metalloproteinases," Biochemical and Biophysical Research Communications, vol. 339, no. 1, pp. 47-54, 2006.

[62] E. M. M. Ooi, D. T. Chan, G. F. Watts et al., "Plasma apolipoprotein C-III metabolism in patients with chronic kidney disease," Journal of Lipid Research, vol. 52, no. 4, pp. 794-800, 2011.

[63] D. T. Chan, G. K. Dogra, A. B. Irish et al., "Chronic kidney disease delays VLDL-apoB-100 particle catabolism: potential role of apolipoprotein C-III," Journal of Lipid Research, vol. 50, no. 12, pp. 2524-2531, 2009.

[64] M. A. Navarro, R. Carpintero, S. Acín et al., "Immune-regulation of the apolipoprotein A-I/C-III/A-IV gene cluster in experimental inflammation," Cytokine, vol. 31, no. 1, pp. 52-63, 2005.

[65] M. Riwanto and U. Landmesser, "High density lipoproteins and endothelial functions: mechanistic insights and alterations in cardiovascular disease," Journal of Lipid Research, vol. 54, no. 12, pp. 3227-3243, 2013.

[66] M. Morena, J.-P. Cristol, T. Dantoine, M.-A. Carbonneau, B. Descomps, and B. Canaud, "Protective effects of high-density lipoprotein against oxidative stress are impaired in haemodialysis patients," Nephrology Dialysis Transplantation, vol. 15, no. 3, pp. 389-395, 2000.

[67] V. Witko-Sarsat, V. Gausson, and B. Descamps-Latscha, "Are advanced oxidation protein products potential uremic toxins," Kidney International Supplement, vol. 63, no. 84, pp. S11-S14, 2003.

[68] C. Delporte, P. Van Antwerpen, L. Vanhamme, T. Roumeguère, and K. Zouaoui Boudjeltia, "Low-density lipoprotein modified by myeloperoxidase in inflammatory pathways and clinical studies," Mediators of Inflammation, vol. 2013, Article ID 971579, 18 pages, 2013.

[69] S. Miyamoto, G. R. Martinez, D. Rettori, O. Augusto, M. H. G. Medeiros, and P. Di Mascio, "Linoleic acid hydroperoxide reacts with hypochlorous acid, generating peroxyl radical intermediates and singlet molecular oxygen," Proceedings of the National Academy of Sciences of the United States of America, vol. 103, no. 2, pp. 293-298, 2006. 
[70] F. O. Ismael, J. M. Proudfoot, B. E. Brown et al., “Comparative reactivity of the myeloperoxidase-derived oxidants $\mathrm{HOCl}$ and HOSCN with low-density lipoprotein (LDL): implications for foam cell formation in atherosclerosis," Archives of Biochemistry and Biophysics, vol. 573, pp. 40-51, 2015.

[71] E. Malle, G. Marsche, J. Arnhold, and M. J. Davies, "Modification of low-density lipoprotein by myeloperoxidase-derived oxidants and reagent hypochlorous acid," Biochimica et Biophysica Acta, vol. 1761, no. 4, pp. 392-415, 2006.

[72] D. M. Okamura, J. M. López-Guisa, K. Koelsch, S. Collins, and A. A. Eddy, "Atherogenic scavenger receptor modulation in the tubulointerstitium in response to chronic renal injury," American Journal of Physiology-Renal Physiology, vol. 293, no. 2, pp. F575-F585, 2007.

[73] E. U. Rahman, X. Z. Ruan, R. S. Chana et al., "Mesangial matrixactivated monocytes express functional scavenger receptors and accumulate intracellular lipid," Nephrology Dialysis Transplantation, vol. 23, no. 6, pp. 1876-1885, 2008.

[74] K.-A. Rye, "Biomarkers associated with high-density lipoproteins in atherosclerotic kidney disease," Clinical and Experimental Nephrology, vol. 18, no. 2, pp. 247-250, 2014.

[75] A. Bakillah, F. Tedla, I. Ayoub et al., "Plasma nitration of highdensity and low-density lipoproteins in chronic kidney disease patients receiving kidney transplants," Mediators of Inflammation, vol. 2015, Article ID 352356, 11 pages, 2015.

[76] B. Shao, C. Bergt, X. Fu et al., "Tyrosine 192 in apolipoprotein A-I is the major site of nitration and chlorination by myeloperoxidase, but only chlorination markedly impairs ABCA1dependent cholesterol transport," Journal of Biological Chemistry, vol. 280, no. 7, pp. 5983-5993, 2005.

[77] S. Yamamoto, P. G. Yancey, T. A. Ikizler et al., "Dysfunctional high-density lipoprotein in patients on chronic hemodialysis," Journal of the American College of Cardiology, vol. 60, no. 23, pp. 2372-2379, 2012.

[78] N. D. Vaziri, "Dyslipidemia of chronic renal failure: the nature, mechanisms, and potential consequences," American Journal of Physiology-Renal Physiology, vol. 290, no. 2, pp. F262-F272, 2006.

[79] H. Kimura, R. Miyazaki, T. Imura et al., "Hepatic lipase mutation may reduce vascular disease prevalence in hemodialysis patients with high CETP levels," Kidney International, vol. 64, no. 5, pp. 1829-1837, 2003.

[80] S. A. Saeed, M. Elsharkawy, K. Elsaeed, and O. Fooda, "Paraoxonase-1 (PON1) activity as a risk factor for atherosclerosis in chronic renal failure patients," Hemodialysis International, vol. 12, no. 4, pp. 471-479, 2008.

[81] H. Honda, M. Ueda, S. Kojima et al., "Oxidized high-density lipoprotein is associated with protein-energy wasting in maintenance hemodialysis patients," Clinical Journal of the American Society of Nephrology, vol. 5, no. 6, pp. 1021-1028, 2010.

[82] E. D. Van Deel, D. Merkus, R. Van Haperen, M. C. de Waard, R. de Crom, and D. J. Duncker, "Vasomotor control in mice overexpressing human endothelial nitric oxide synthase," American Journal of Physiology-Heart and Circulatory Physiology, vol. 293, no. 2, pp. H1144-H1153, 2007.

[83] D. C. Abrantes, T. M. C. Brunini, C. Matsuura et al., "Diminished nitric oxide generation from neutrophils suppresses platelet activation in chronic renal failure," Molecular and Cellular Biochemistry, vol. 401, no. 1-2, pp. 147-153, 2014.

[84] J. Himmelfarb, "Uremic toxicity, oxidative stress, and hemodialysis as renal replacement therapy," Seminars in Dialysis, vol. 22, no. 6, pp. 636-643, 2009.
[85] S. Galijasevic, G. M. Saed, M. P. Diamond, and H. M. AbuSoud, "Myeloperoxidase up-regulates the catalytic activity of inducible nitric oxide synthase by preventing nitric oxide feedback inhibition," Proceedings of the National Academy of Sciences of the United States of America, vol. 100, no. 25, pp. 14766-14771, 2003.

[86] P. Stenvinkel, J. J. Carrero, J. Axelsson, B. Lindholm, O. Heimbürger, and Z. Massy, "Emerging biomarkers for evaluating cardiovascular risk in the chronic kidney disease patient: how do new pieces fit into the uremic puzzle?" Clinical Journal of the American Society of Nephrology, vol. 3, no. 2, pp. 505-521, 2008.

[87] W. K. C. Lai and M. Y. Kan, "Homocysteine-induced endothelial dysfunction," Annals of Nutrition and Metabolism, vol. 67, no. 1, pp. 1-12, 2015.

[88] R. H. Böger, S. M. Bode-Böger, P. S. Tsao, P. S. Lin, J. R. Chan, and J. P. Cooke, "An endogenous inhibitor of nitric oxide synthase regulates endothelial adhesiveness for monocytes," Journal of the American College of Cardiology, vol. 36, no. 7, pp. 22872295, 2000.

[89] G. Tripepi, F. M. Raso, E. Sijbrands et al., "Inflammation and asymmetric dimethylarginine for predicting death and cardiovascular events in ESRD patients," Clinical Journal of the American Society of Nephrology, vol. 6, no. 7, pp. 1714-1721, 2011.

[90] S. Abedini, A. Meinitzer, I. Holme et al., "Asymmetrical dimethylarginine is associated with renal and cardiovascular outcomes and all-cause mortality in renal transplant recipients," Kidney International, vol. 77, no. 1, pp. 44-50, 2010.

[91] A. A. Mangoni, C. L. Hewitson, R. J. Woodman, M. J. Whiting, B. McAteer-Carr, and J. A. Barbara, "Symmetric dimethylarginine is an independent predictor of intradialytic hypotension," American Journal of Hypertension, vol. 21, no. 8, pp. 955-959, 2008.

[92] E. Schepers, G. Glorieux, A. Dhondt, L. Leybaert, and R. Vanholder, "Role of symmetric dimethylarginine in vascular damage by increasing ROS via store-operated calcium influx in monocytes," Nephrology Dialysis Transplantation, vol. 24, no. 5, pp. 1429-1435, 2009. 


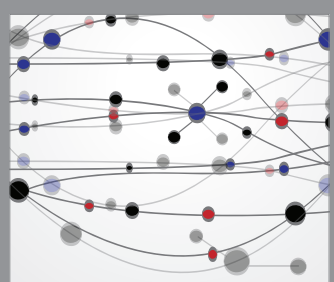

The Scientific World Journal
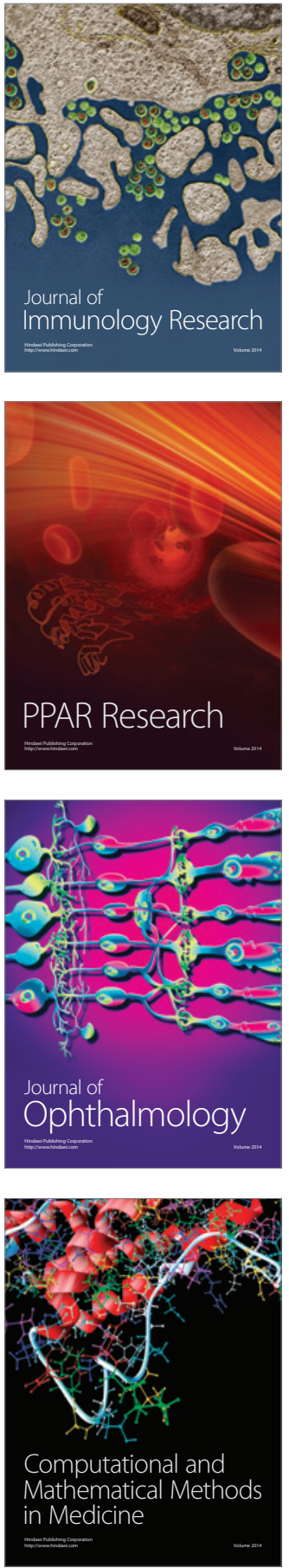

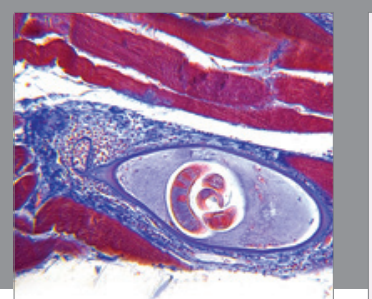

Gastroenterology Research and Practice

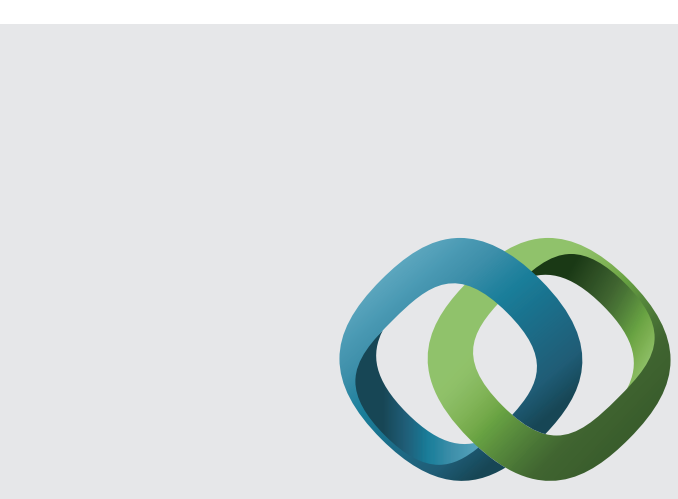

\section{Hindawi}

Submit your manuscripts at

http://www.hindawi.com
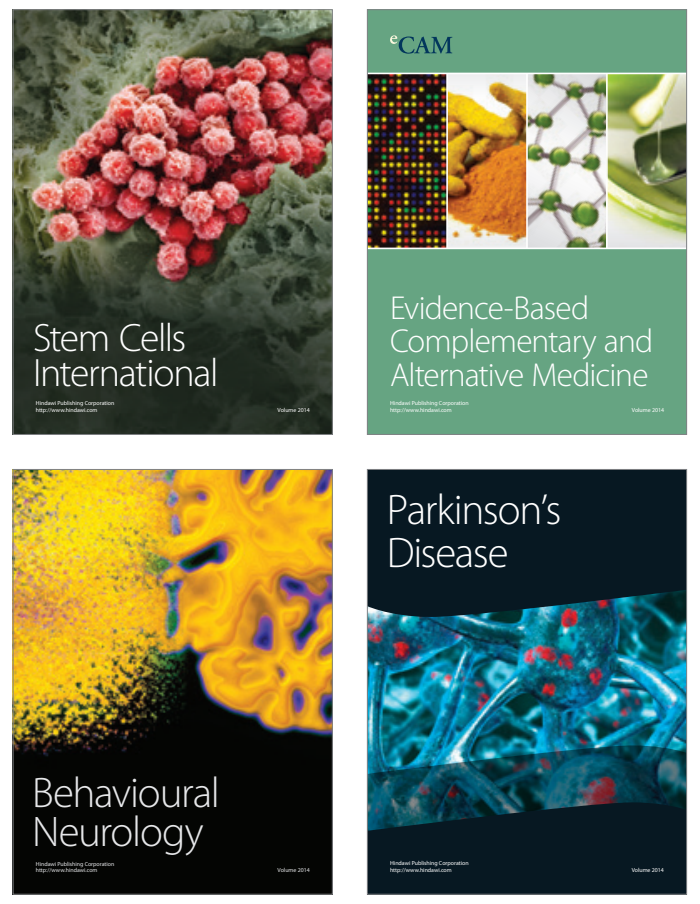
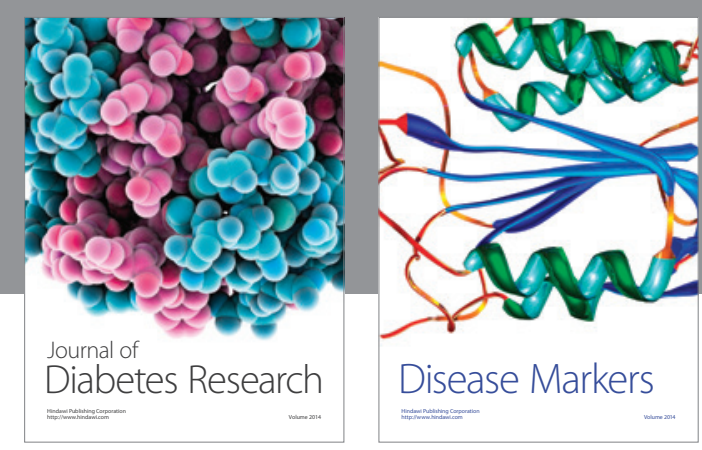

Disease Markers
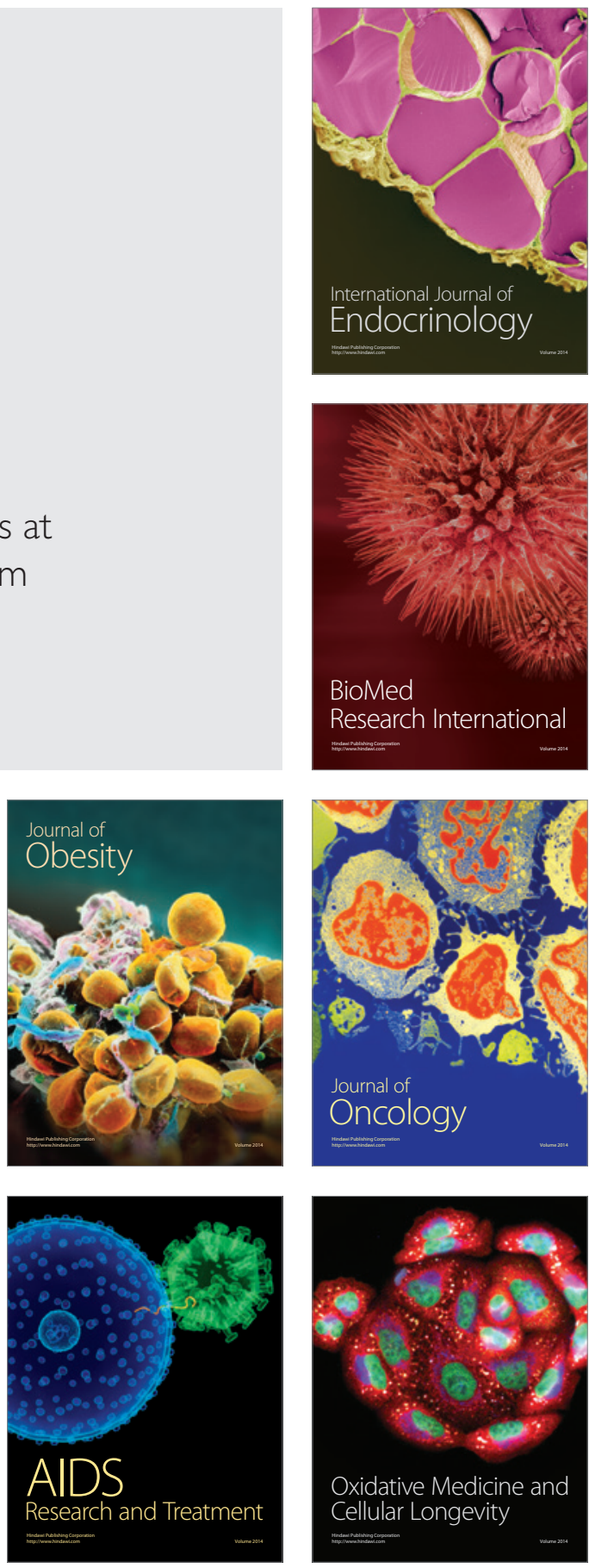